\title{
HYBRID AMPLIFIER MODULES FOR NUCLEAR MEASUREMENTS IN SPACE FLIGHT EXPERIMENTS $\dagger$
}

\author{
WALTER FUNK ${ }^{\ddagger}$ and WOLFGANG WINKELNKEMPER ${ }^{\S}$ \\ $¥$ Philips GmbH Forschungslaboratorium Hamburg, D-2000 Hamburg 54, FRG \\ $\S$ Ruhr-Universität Bochum, Institut für Elektronik, Postfach 102148 D-4630 Bochum 1, FRG
}

(Received June 16, 1979)

\begin{abstract}
Nuclear electronic instruments for the measurement of the precise energy and time-of-flight of charged particles, e.g. heavy ions, are quite of ten present on scientific satellites. In addition to the non-trivial electrical requirements, size, weight, and power consumption have to be kept within strict limits for space flight electronics. Furthermore, such instruments must possess a wide operating temperature range and resistance to irradiation. In the interest of cost effectiveness it is desirable to develop modules with a sufficiently wide application to enable their use on more than one mission. Because of the small quantities (expected need is in the order of some hundred circuits per year) and the resistance and capacitance values required, a hybrid thick film approach is considered to be the most economical solution. The development of two amplifier modules took place in a co-operation between the Max-Planck-Institut für Aeronomie in Lindau/Harz (scientific and environmental requirements), the Hahn-Meitner-Institut Berlin (irradiation treatment), the Lehrstuhl für Elektronische Schaltungen of the Ruhr-Universität Bochum (electrical design), and the Philips Forschungslaboratorium Hamburg (technological implementation and production of prototypes). The results and experiences are reported in this paper.
\end{abstract}

\section{INTRODUCTION}

The analogue part of a typical set-up for the energy measurements of charged particles is shown in Figure 1.

An incoming particle creates charge carriers within a reverse biased detector diode $(H V=$ high voltage, typically a few volts to several hundred volts). The total amount of charge is proportional to the energy, $E_{D E P}$, received by the detector. The diode is connected to the summing point of an integrator, the charge therefore is transferred to and stored on the

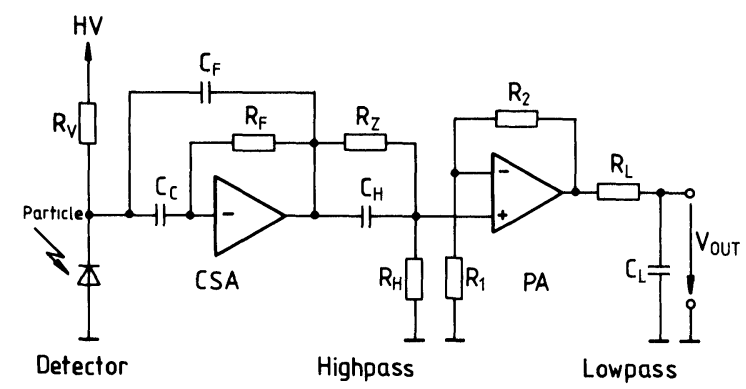

FIGURE 1 Analog Part of the Energy Measurement Set-up.

†The described work was sponsored by the German Federal Ministry for Research and Technology (BMFT). Only the authors are responsible for the contents of this publication. feedback capacitance $C_{F}$ (typically $1 p F$ ). Thus the amplifier output voltage is proportional to the charge ("Charge Sensitive Amplifier", CSA). The capacitor $C_{C}$ protects the amplifier from high voltage and can be seen as a signal short circuit, $R_{F}$ is a bias resistor of several hundred $M \Omega$. Typical design objectives of the CSA include:

- dc-voltage gains in excess of $80 \mathrm{~dB}$,

- unity-gain frequency up to several hundred $\mathrm{MHz}$,

- stable operation at closed-loop gains down to $20 \mathrm{~dB}$,

- input referred noise voltage spectral density around $1 \mathrm{nV} / \sqrt{\mathrm{Hz}}$.

The CSA output signal is processed through pulse forming networks and amplified in a pulse amplifier (PA). In the simplest form, pulse forming is achieved by a high pass network $\left(C_{H}, R_{H}\right)$ with bypassing resistor $R_{Z}$ to eliminate the differentiating influence of $R_{F}$, followed by a decoupled lowpass network $\left(R_{L}, C_{L}\right)$ as shown in Figure 1. More sophisticated networks yield better signal-to-noise ratios.

The $P A$ is a wideband operational amplifier particularly designed for pulse amplification. Rise and fall times down to and below $10 \mathrm{~ns}$ at closed-loop gains of up to $30 \mathrm{~dB}$ are typical requirements. For 
more details of this measurement technique see e.g. Kowalski ${ }^{1}$ and literature given there.

\section{ELECTRICAL DESIGN}

The influence of irradiation on electronic components has been investigated and reported previously. $^{2,3}$ The information of value to this project can very roughly be summarized as follows (irradiation dose up to $500 \mathrm{krad} S_{i}$ ):

- leakage currents in $p n$-junctions as well as in dielectric materials of insulators and capacitors are increased by an order of magnitude,

- noise currents and voltages are increased,

- current gain $h_{F E}$ of bipolar transistors is decreased dramatically, especially at low collector currents,

- transconductance of JFETs is changed very little,

- resistance and capacitance of passive

components is almost unaffected.

This information could be confirmed by measurements carried out before the amplifiers were designed. Some measurements on thin- and thick-film resistors show no significant difference in comparison to discrete carbon or metal film resistors.

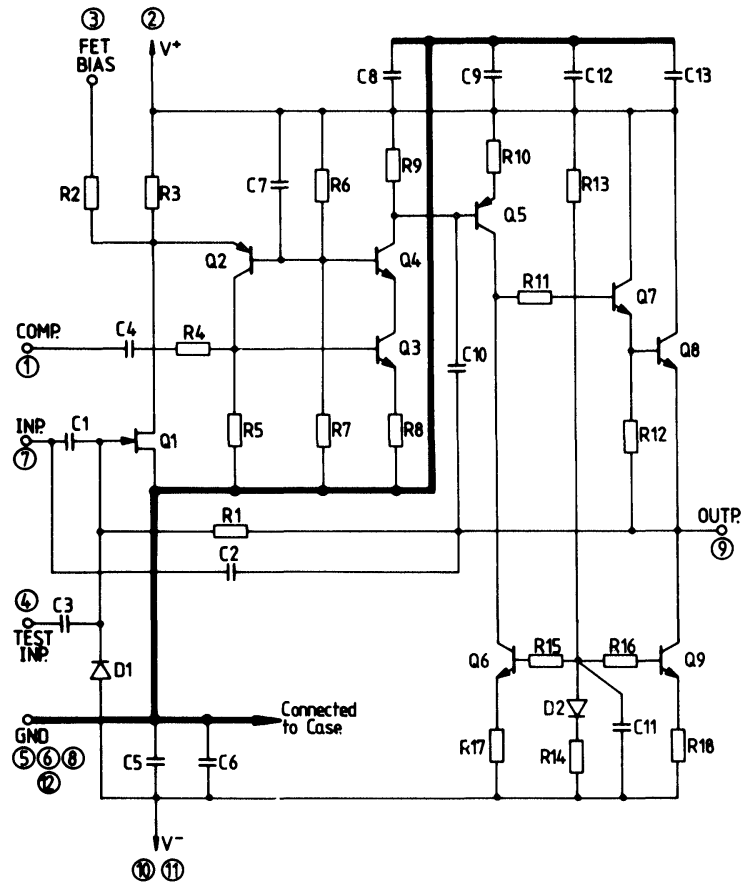

FIGURE 2 Schematic of the CSA.

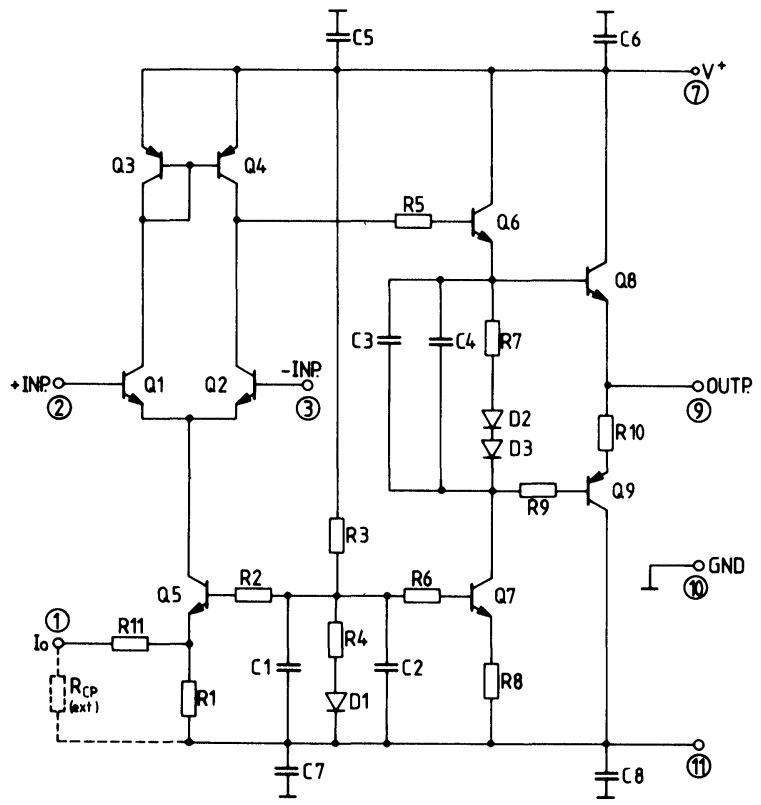

FIGURE 3 Schematic of the $P A$.

From these topics the main design criterion can be readily defined: The circuits must be highly insensitive to $h_{F E}$-degradation of the transistors (which, simultaneously, must be valid for a wide operating temperature range).

The schematics of the two amplifiers are shown in Figure $2(C S A)$ and Figure $3(P A)$. The above design rule is realized by individual feedback per stage $\left(R_{8}, R_{10}, C_{10}\right.$ in the $\left.C S A\right)$ and Darlington-connected output stages in both amplifiers.

The $C S A$ is composed of three cascaded voltage gain stages yielding very high gain reserves for a wide field of applications. Frequency compensation is accomplished with an external resistor from "Comp" to Ground. More details of the circuit will be reported elsewhere. ${ }^{4}$

The $P A$ is a very simple approach, allowing the current of the differential state and therefore the open-loop gain to be programmed with an external resistor, $R_{C P}$.

\section{PHYSICAL DESIGN AND IMPLEMENTATION}

Space flight instruments are exposed to high acceleration forces and irradiation. Light weight and small size are essential. The expected demand of a few hundred circuits would require a hybrid solution in thin film technology. The requirement of resistors 
in the range $22 \Omega$ to $55 \mathrm{k} \Omega$ and of the smallest substrate size possible, as well as the high frequency range envisaged, excludes meandering of the resistors. Thick film technology was therefore the only choice. To guarantee high resistor stability an iridium-based resistor paste, fireable at high temperatures was chosen and a chip was used for the $500 \mathrm{M} \Omega$ resistor.

As the non-differential structure of the CSA has a poor power supply rejection, voltage drops in the conductor lines due to the output current are intolerable. In addition to having only one bonding technique (reflow soldering), we have chosen a high temperature fireable $P T / A U$ paste for the conductor pattern. The high firing temperature of $995^{\circ} \mathrm{C}$ resulted in a good conductivity, excellent adhesion to the $\mathrm{Al}_{2} \mathrm{O}_{3}$ substrate and negligible solder leaching.

To avoid parasitic capacitances (even a few pico-farad impair the circuit properties and the high value of $\epsilon_{r}$ for the $\mathrm{Al}_{2} \mathrm{O}_{3}$ substrate is a limitation one has to live with), we paid special attention to the design of the layouts. This together with several wire bonds between the ground plane and the metal case resulted in the just tolerable addition of $1 \mathrm{pF}$ to the $3 \mathrm{pF}$ between the collectors of $Q 2$ and $Q 4$ of the
$P A$ and approximately $0.4 \mathrm{pF}$ in parallel to the feedback $C_{2}(1 \mathrm{pF})$ with a $T K \approx 300 \mathrm{ppm} /{ }^{\circ} \mathrm{C}$.

Unfortunately, not all the necessary active devices were available in such a suitable package as the SOT 23 and so some standard transistor packages had to be used. The necessary capacitances could be realized by existing multilayer ceramic chip capacitors.

\section{MEASURED PERFORMANCE}

The measured frequency responses are shown in Figure $4(C S A)$ and Figure $5(P A)$. The gain-bandwidth product of the $C S A$ with $R=1 \mathrm{k} \Omega$ compensation is about $1.6 \mathrm{GHz}$ at $50 \mathrm{MHz}(3 \mathrm{GHz}$ uncompensated, but with more phase lag, i.e. not suitable for closedloop operation at gains below $40 \mathrm{~dB}$ ). The influence of the compensation resistor is obvious. The step response of the $C S A$ and its variation with temperature is shown in Figure 6 (conditions: $40 \mathrm{~dB}$ gain, $C S A$ properly compensated, load impedance $100 \Omega$ ).

The $P A$ has a $600 \mathrm{MHz}$ gain-bandwid th product (approx) at $50 \mathrm{MHz}$ with $20 \mathrm{~mW}$ power consumption (300 MHz with $13.5 \mathrm{~mW}, 140 \mathrm{MHz}$ with $11 \mathrm{~mW}$ ). The

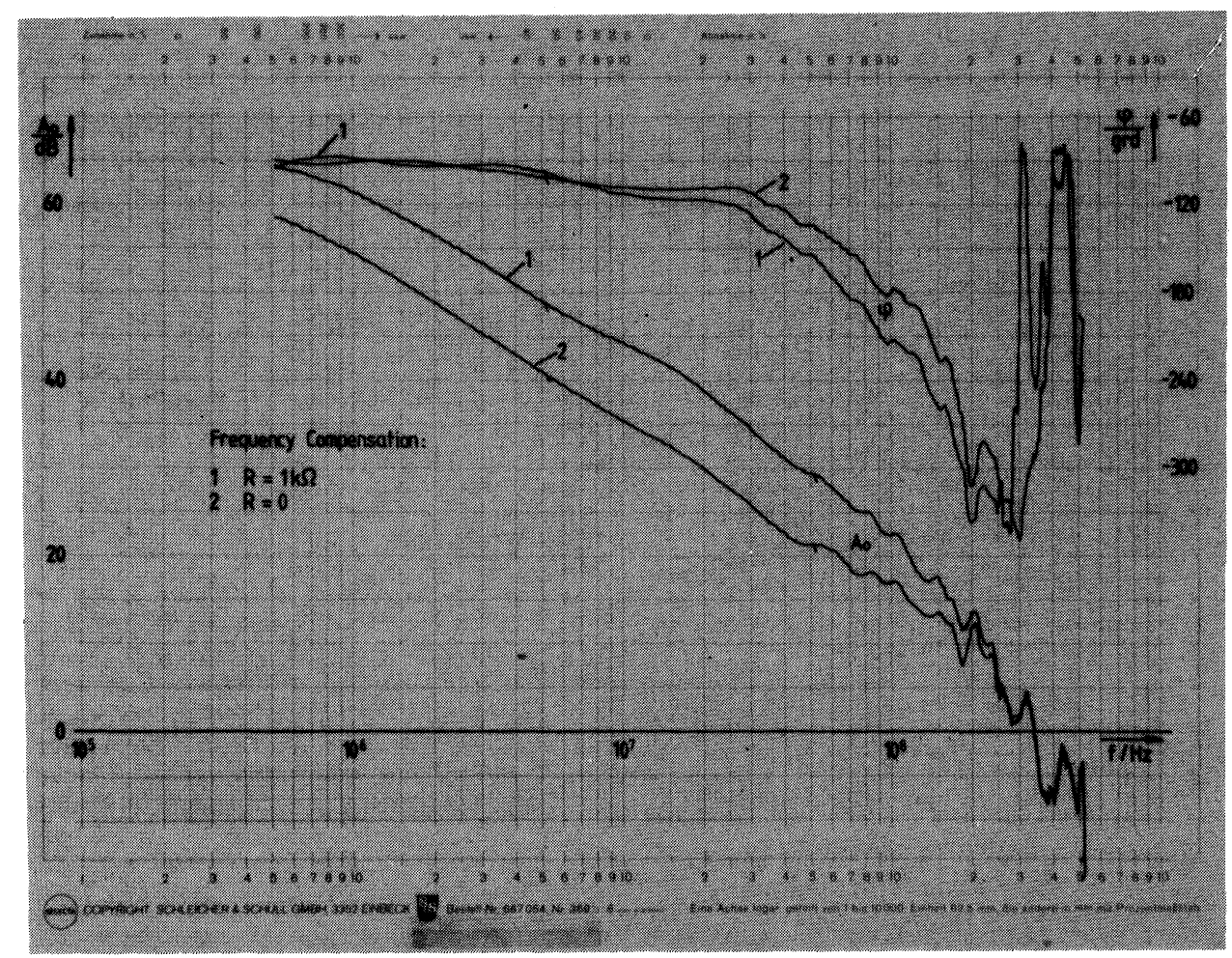

FIGURE 4 Frequency Response of the $C S A$. 


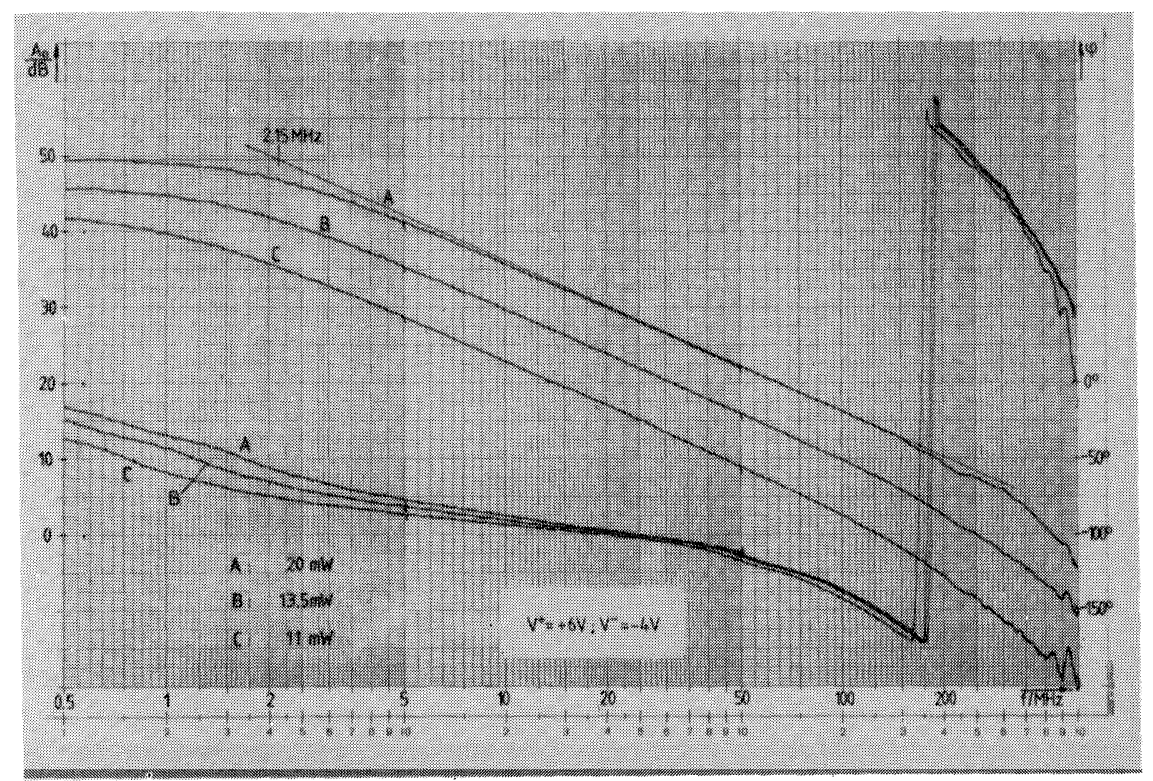

FIGURE 5 Frequency Response of the $P A$.

dynamic performance only varied within the accuracy of measurement on exposure to ${ }^{60} \mathrm{Co} \gamma$-irradiation to a total dose of $200 \mathrm{krad} \mathrm{Si}$. The noise behaviour, however, was degraded exactly as was to be expected from the measurements on the semiconductors used in the input stage.

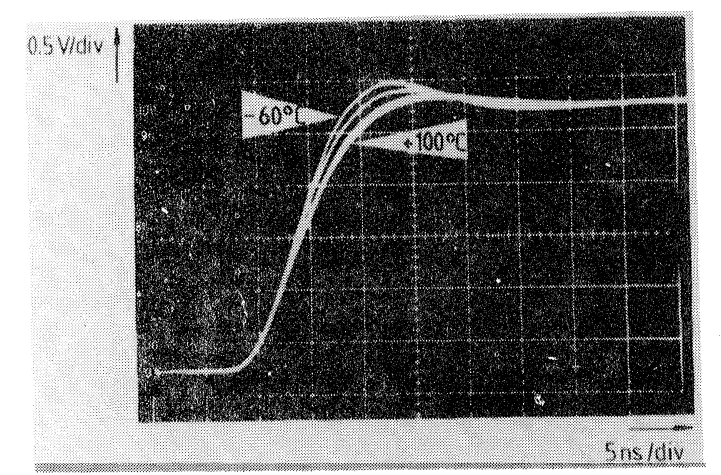

FIGURE 6 Step response variation over temperature of the CSA.

\section{CONCLUSIONS}

Despite the unavoidable bandwidth degradation, resulting from the use of hybrid thick film technology with ceramic $\mathrm{Al}_{2} \mathrm{O}_{3}$-substrates in low-power wideband operational amplifiers, the parasitic capacitances could be kept low enough to achieve reasonable performance. Reliability considerations will probably compel a new layout with chip transistors instead of plastic encapsulated ones. No fundamentally new problems are to be expected in this step. We think that hybrid thick film technology is a good compromise between physical size, electrical performance, and cost for space flight applications.

\section{ACKNOWLEDGEMENT}

The authors are indebted to J. W. Klein for his encouragement and competent advice, and D. Bräuning for his friendly support in irradiation questions.

\section{REFERENCES}

1. E. Kowalski, Nuclear Electronics, Springer-Verlag 1970.

2. A. Spencker, H. G. Wagemann, D. Bräuning, Strahlungsbelastungs-Untersuchungen an elektronischen Bauelementen des SYMPHONIE-Satelliten, Report HMI-B 181 (Sept. 1975), Hahn-Meitner-Institut, Berlin.

3. A. G. Stanley, K. E. Martin, S. Douglas, Radiation Design Criteria Handbook. Technical Memorandum 33-763, Jet Propulsion Laboratory (Aug. 1976), Pasadena, Cal.

4. J. R. Cessna, A. Glasmachers, W. Winkelnkemper, An Investigation of Fast Low Power Charge Sensitive Preamplifier Design, IEEE Trans. Vol. NS-27, No. 1, Feb. 1980, pp. 308-312. 

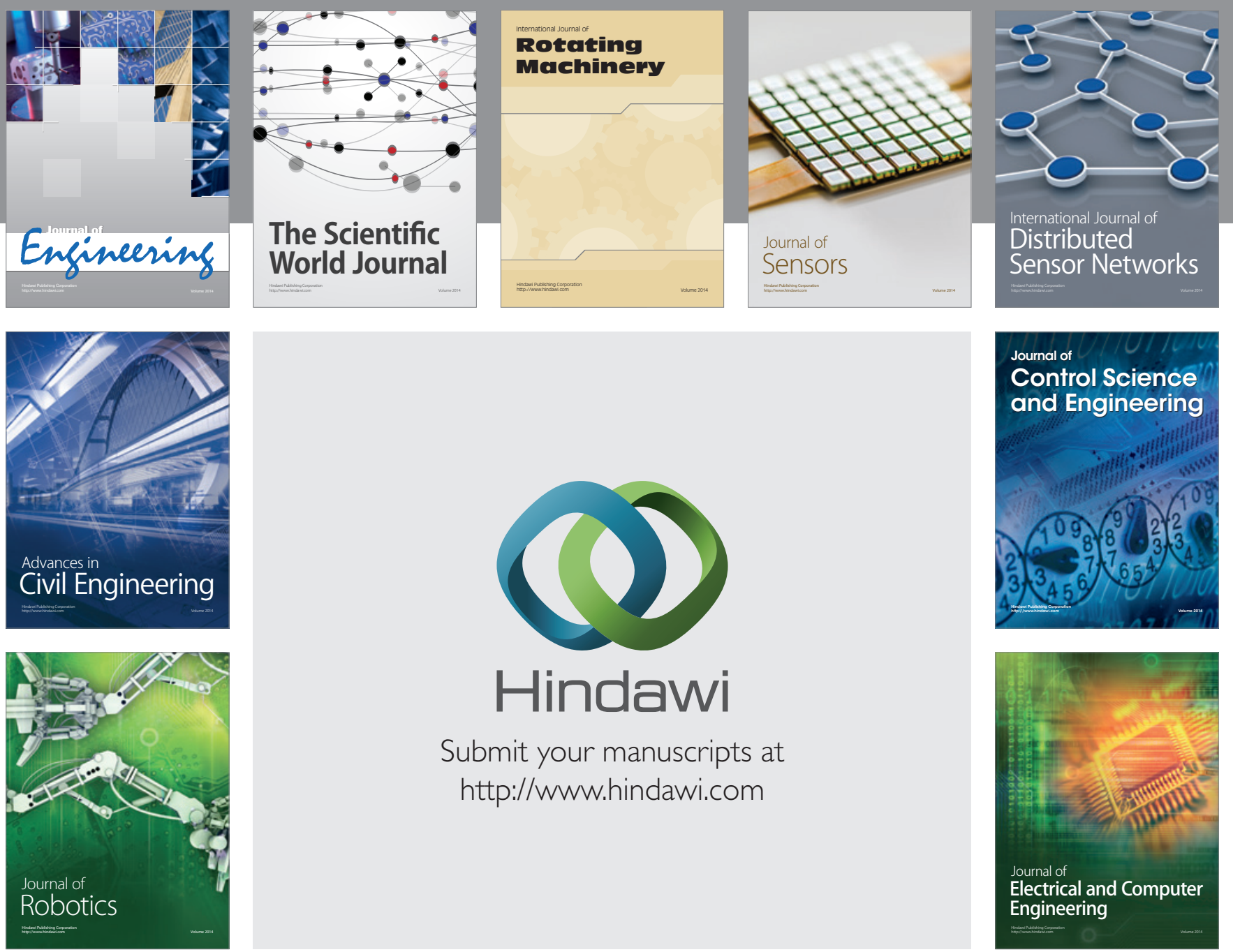

Submit your manuscripts at

http://www.hindawi.com
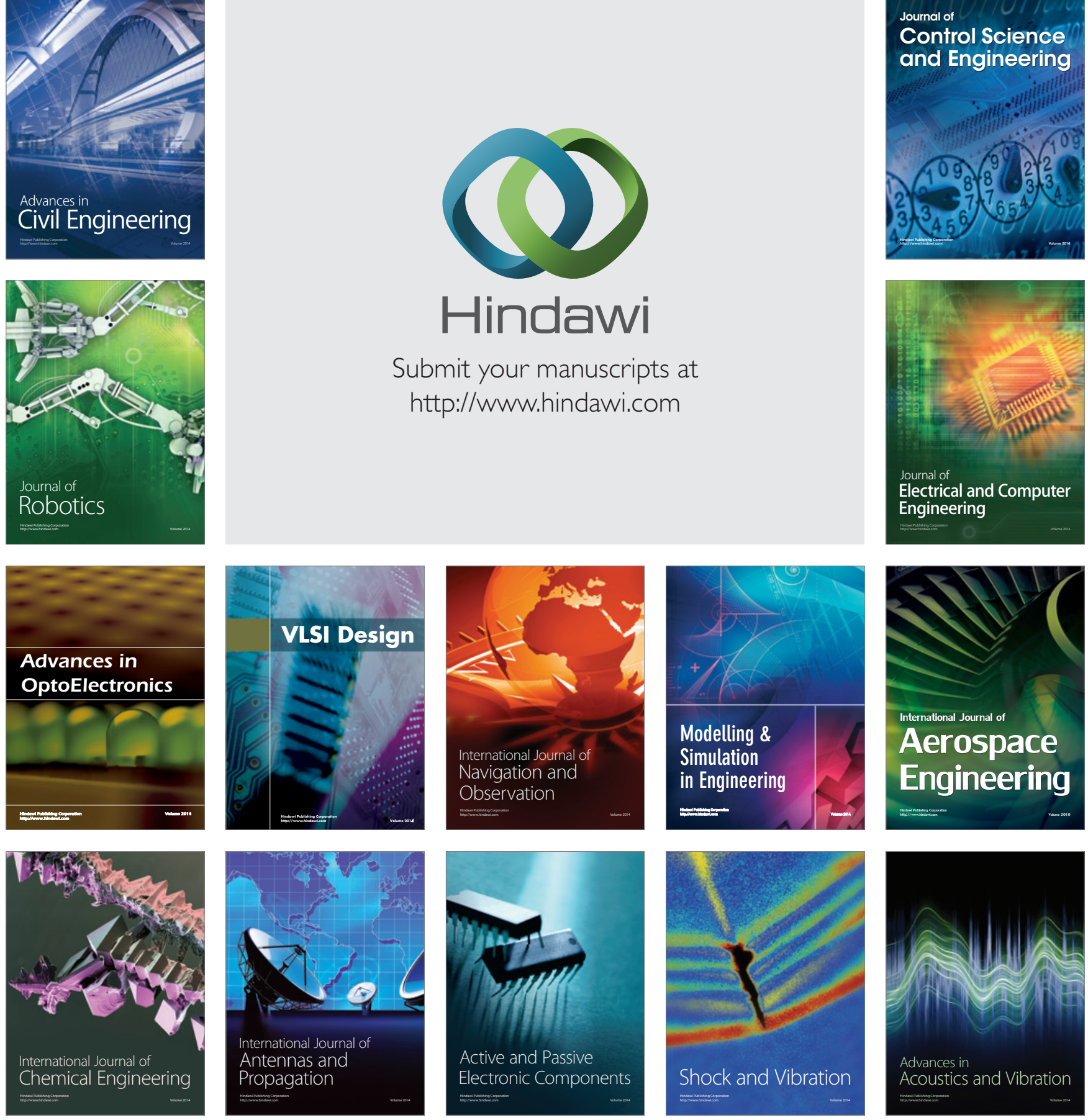